\title{
Channel Gain for a Wrist-to-Arm Scenario in the 55- 65 GHz Frequency Band
}

\author{
Arno Thielens ${ }^{1,2}$, Reza Aminzadeh ${ }^{2}$, Luc Martens², Wout Joseph², and Jan Rabaey ${ }^{1}$ \\ ${ }^{1}$ Berkeley Wireless Research Center, Department of Electrical Engineering and \\ Computer Sciences, University of California Berkeley \\ Berkeley, CA 94704, USA \\ ${ }^{2}$ Department of Information Technology, Ghent University, IMEC, 9052 Ghent, Belgium \\ arno.thielens@ugent. be
}

\begin{abstract}
Wireless communication on the body is expected to become more important in the future. This communication will in certain scenarios benefit from higher frequencies of operation and their associated smaller antennas and potentially higher bandwidths. One of these scenarios is communication between a wristband and wearable sensors on the arm. In order to investigate the feasibility of such a scenario, propagation at $55-65 \mathrm{GHz}$ along the arm is measured for two configurations. First, for increasing separation distances along the arm, and second for a transmitter is rotationally placed around the wrist. Two channel gain models are fitted to the data and used to obtain a channel gain exponent in the first configuration and loss per angle of rotation in the second configuration. These models are relevant inputs for the design of future wearable wireless systems.
\end{abstract}

Keywords: Body Area Networks, 5G Communication, mm-wave Propagation.

\section{Introduction}

There is a growing interest in Body Area Networks (BANs). These collections of wireless, body-worn sensors and actuators are envisioned to enable a large amount of wearable applications [1,2]. A standard for wireless communication in BANs (IEEE 802.15.6) [3] has been developed and several applications already exist [1,2]. There are three types of communication within a BAN: on-body communication between two body-worn nodes, off-body communication between a body-worn node, and body surface to intra-body communication between a body-worn node and an implanted node [3]. The current generation of BANs mainly operate below $6 \mathrm{GHz}$. However, there is a trend in wireless research, for example in $5^{\text {th }}$ generation networks, to consider higher frequency bands. One of these potential frequency bands is the V-band $(40-70 \mathrm{GHz})$, where a license-free frequency band is allocated around $60 \mathrm{GHz}(57-64 \mathrm{GHz}$ in the US). The advantages of using this relatively high frequency band is the use of smaller antenna (arrays) and higher bandwidths. Two aspects that are very appealing for BANs as well. 
An application that could benefit greatly from $60 \mathrm{GHz}$ communication is the wristband to arm scenario illustrated in Fig. 1. It is conceivable that a wristband (of for example a smart watch) contains multiple interconnected antennas that work at $60 \mathrm{GHz}$ and cover an arm with several scattered wireless sensors, for example: Electromyography (EMG), skin impedance, blood oxygenation, and blood oximetry sensors that are not necessarily all on the same location. Using the right protocol and antenna combiners, such a wristband array could be used for on-body beam steering [17] or Multiple Input Multiple Output (MiMo) communication [18]. This will provide premium channel gains and signal-to-noise and signal-to-interference ratios. In order to enable such a network, the propagation along the arm needs to be studied first. In particular, the channel loss or equivalently gain $[4,16]$ that can exist between such nodes.

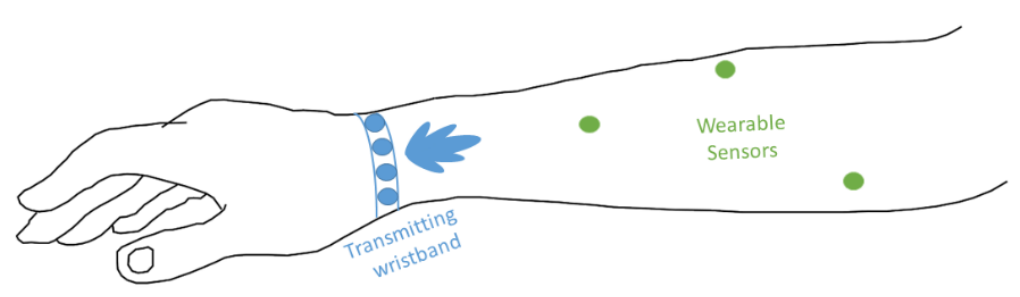

Fig. 1. Illustration of the concept of a wristband with multiple $60 \mathrm{GHz}$ transmitters communicating with several wearable sensors on the arm.

There have been previous studies of on-body antennas at $60 \mathrm{GHz}$ [5-7] and on-body channel loss measurements at $60 \mathrm{GHz}$ [5, 8-10]. However, the number of studies is limited and are either based on measurements and simulation using phantoms $[5,8,10]$ or only consider certain fixed links (fixed transmitter (TX) and receiver (RX)) on the human body such as "shoulder to ankle", "wrist to head" [5,9] and do not provide insight into the relationship between channel loss and propagation distance. We did not find previous publications that study propagation along the arm at $60 \mathrm{GHz}$. Therefore, the goals of this study were the following: (1) execute channel gain measurements at $60 \mathrm{GHz}$ as function of distance along the arm of real humans, (2) execute channel gain measurements for rotated TX antennas on the wrist that emulate a wristband with multiple antennas, and (3) develop a channel gain model for propagation in that scenario.

\section{$2 \quad$ Materials and Methods}

\subsection{Theory}

Propagation of vertically (V) and horizontally $(\mathrm{H})$ polarized electromagnetic fields on a flat conductive surface has been described in literature $[11,12]$ and more specific at $60 \mathrm{GHz}$ in [8]. For a V-polarized wave, the electric field $E_{r}$ at the location of a receiver at a height of $h_{r}$ and distance $d$ from a transmitter at height $h_{T}$, can be expressed (near the surface) as $[8,11,12]$ : 


$$
E_{r}=E_{Q S}+E_{\text {geom }}+E_{\text {surf }}
$$

with $E_{r}$ the electric field at the receiver, $E_{Q S}$ is the quasi-static field, $E_{\text {geom }}$ is the geometrical-optics field, and $E_{\text {surf }}$ is the Norton Surface wave. The relative amplitudes of the last two terms depend on $\mathrm{d}, \mathrm{h}_{\mathrm{r}}, \mathrm{h}_{\mathrm{T}}$, the dielectric parameters of the conductive surface, and the transmitter and receiver parameters. Expressions for both terms can be found in $[8,11,12]$. The validity of this approximation is discussed in [8] and requires that $h_{T}$ and $h_{R}$ are maximally a couple of millimeters. The first term in Eq. 1 governs the near-field transmission (quasi-static coupling) and has a $1 / d^{3}$ dependency. At $60 \mathrm{GHz}$, this component is small at propagation distances of several centimeters [8]. The second and third terms will be the dominant in the channel measurements executed in this study (see Section 2.2). Both terms have a $1 / d^{2}$ dependency [8], which has led to the proposal of the following channel gain/loss model in [8]:

$$
G(d)=G\left(d_{0}\right)-10 \cdot n \cdot \log _{10}\left(\frac{d}{d_{0}}\right)+X_{\sigma}
$$

with $\mathrm{G}$ the channel gain in decibels, $\mathrm{d}_{0}$ an arbitrary reference distance, $n$ the channel gain exponent, and $X_{\sigma}$ the lognormal variance on the pathloss. As [8] proposes a $1 / d^{2}$ dependency of the surface waves, they predicted and found an $n=4$ for the channel loss, which scales as $E_{r}^{2}$.

Another analysis can be done for H-polarized waves, leading to a similar channel gain model [8]. Therefore, this model will be used in Section 2.3 to process the measurement data.

The dependencies of channel gain over distance assume perfect alignment in terms of polarization. However, in the case of a wristband containing several transmitting antennas, there will be a polarization mismatch between TX and RX. Moreover, the field will propagate along a curved surface instead of an approximately straight path. The polarization mismatch between two linearly-polarized antennas can be described using [13]:

$$
g(\psi)=g\left(0^{\circ}\right) \cdot \cos ^{2}(\psi)
$$

with $\mathrm{g}$ the linear channel gain and $\psi$ the angle between the polarizations of the receiver and the transmitter. This mismatch will decrease channel gain as the TX-RX pair is rotated over angles between $0^{\circ}$ and $90^{\circ}$ relative to one another and increase again for further rotations beyond $90^{\circ}$ up to $180^{\circ}$. Channel gain along a curved surface is commonly described using an exponential dependency [14]:

$$
G(d)=G(0)+10 \cdot \log _{10}\left(e^{-\alpha d}\right)+X_{\sigma}
$$

with $\mathrm{G}$ the channel gain in decibels, $\alpha$ the loss per unit distance, and $X_{\sigma}$ the lognormal variance on the pathloss. Of course, $\mathrm{d}$ is also a function of the angle $\psi$ when two antennas are rotated with respect to one another along the arm. Eqs. 3 and 4 both cause a 
decrease in channel gain between $0^{\circ}$ and $90^{\circ}$ rotation of linearly polarized TX and RX relative to one another. However, while Eq. 4 predicts a further increase in channel gain between $90^{\circ}$ and $180^{\circ}$, Eq. 3 predicts an increase due to a better polarization match. Therefore, an interesting option is the use of a two-slope channel loss model like the one proposed in [15]:

$$
\begin{gathered}
G(\theta)=G\left(0^{\circ}\right)-\alpha_{1} \cdot|\theta| \text { for } \theta \epsilon\left[-\theta_{c},+\theta_{c}\right] \\
G(\theta)=G\left(\theta_{c}\right)-\alpha_{2} \cdot|\theta|-\theta_{c} \text { for } \theta \in\left[-\pi,-\theta_{c}\right] \text { and } \theta \in\left[\theta_{c}, \pi\right]
\end{gathered}
$$

with $\theta$ the angle of rotation around the arm, $\theta_{c}$ the transition angle, and $\alpha_{1}$ and $\alpha_{2}$ the two losses over distance with $\alpha_{1}>\alpha_{2}$.

\subsection{Measurements}

The goal of our measurements was to emulate a wristband for a wearable sensor communication application on the arm. To this aim, channel gain was measured along the arm for two scenarios: (1) transmission along the arm and (2) communication between a rotated transmitter and a fixed receiver, in a frequency band from 55 to $65 \mathrm{GHz}$. The wireless on-body propagation was studied for the scenarios illustrated in Fig. 2.

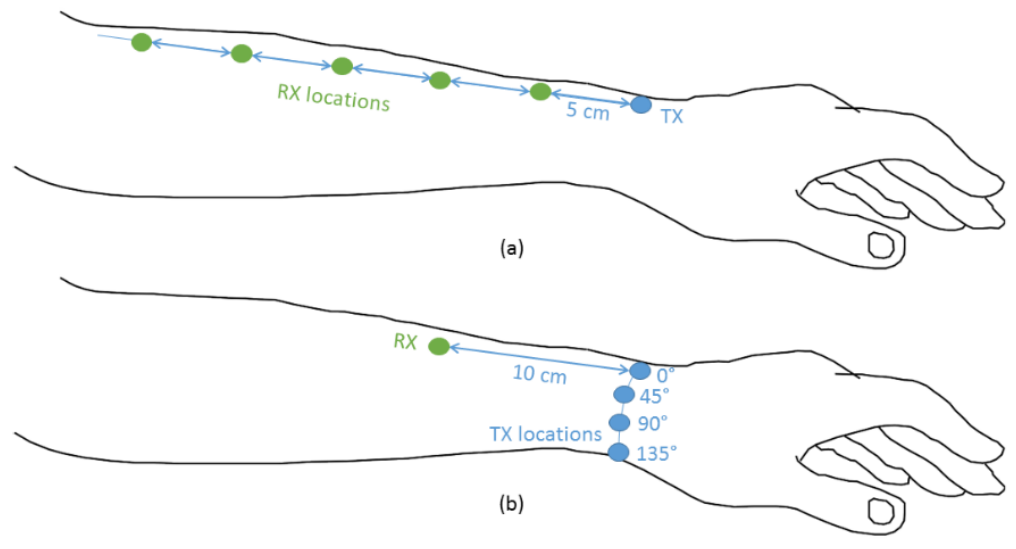

Fig. 2. On-body channel gain measurement scenarios for (a) propagation along the arm and (b) propagation around the arm at fixed separation distance.

This channel gain was measured on the left arm of three male subjects with heights and body masses of $191 \mathrm{~cm}$ and $83 \mathrm{~kg}, 169 \mathrm{~cm}$ and $75 \mathrm{~kg}$, and $172 \mathrm{~cm}$ and $58 \mathrm{~kg}$, respectively.

The procedure for measuring channel gain was as follows (first scenario). Linearlypolarized horn antennas (QMS-00475, Steatite Antennas, UK) resonating in a frequency band around $60 \mathrm{GHz}$ were placed on the left arm of the subjects at a minimum 
separation distance of $5 \mathrm{~cm}$ on the arm of the subject. The TX was always placed on the wrist $\left(\mathrm{h}_{\mathrm{T}}=5 \mathrm{~mm}\right)$, while the $\mathrm{RX}\left(\mathrm{h}_{\mathrm{r}}=5 \mathrm{~mm}\right)$ was moved away from the TX in steps of $5 \mathrm{~cm}$ up to a separation distance of $45 \mathrm{~cm}$, see Fig. 2 (a). Distances are measured in between horn apertures. The horn antennas were placed in two configurations: H-polarization, i.e. polarization parallel to the skin and V-polarization, i.e. polarization orthogonal to the skin. Fig. 3 shows an illustration of the measurement setup on subject 1 .

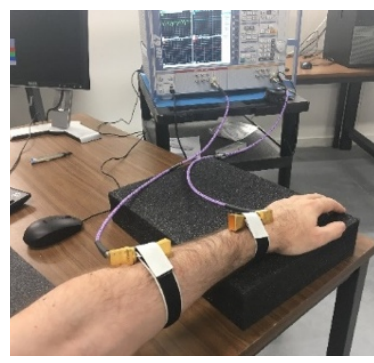

(a)

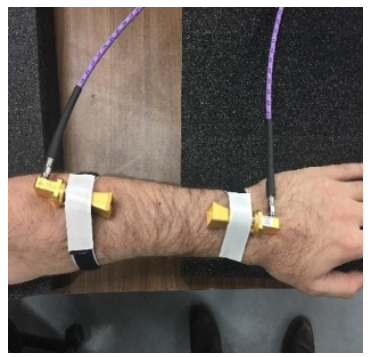

(b)

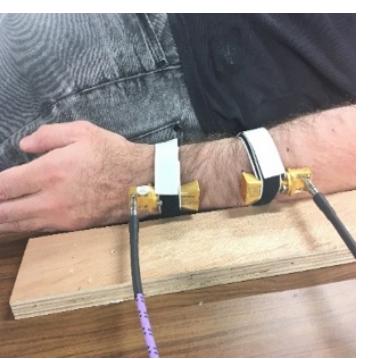

(c)

Fig. 3. Measurement setup on the body of the channel gain measurements (a) and (b) H-configuration, (c) V-configuration.

The horn antennas were connected to a Vector Network Analyzer (ZVA 67, Rohde \& Schwarz, Munich, Germany), which swept over a frequency band from $55-65 \mathrm{GHz}$ in 201 frequency steps. 30 sweeps of all two-port S parameters were registered for every measurement configuration and averaged for processing.

An additional on-body configuration (second scenario) was studied for subject 1 where the TX and RX were aligned and placed at $10 \mathrm{~cm}$ separation distance, see Fig. 2 (b). In this case the TX was again located on the wrist and rotated along the wrist over $360^{\circ}$ in approximate steps of 45 degrees. The RX was static during this rotation. The goal of this measurement is to emulate a wristband containing multiple TX antennas. Both antenna polarizations were again considered in this setup.

\subsection{Data Analysis}

The $S_{21}$ parameter was pooled for every measurement configuration and separation distance, leading to 60 samples per step in distance. These were fed into a log-linear fit using the channel gain model shown in Eq. 2. $\mathrm{d}_{0}$ was chosen to be $10 \mathrm{~cm}$ in this fit. The fit resulted in values for $\mathrm{G}\left(\mathrm{d}_{0}\right)$ and the channel gain exponent $n$. The average deviation $\sigma$ was calculated by averaging the difference (in decibels) between the fitted model and the measurement data.

The $\mathrm{S}_{21}$ and $\mathrm{S}_{12}$ parameters obtained during the rotational measurements were pooled again for every measurement configuration and angle of rotation. Measurements were also pooled for measurements with equal amplitude in rotation angle and opposite sign (for example $45^{\circ}$ and $-45^{\circ}$ were pooled) leading to 120 samples per angle. Based on Eq. 3 we enforced a transition angle of $90^{\circ}$ and fitted two slopes to the measurements at angles $|\theta|<90^{\circ}$ and $|\theta|>90^{\circ}$ in order to obtain $\alpha_{1}$ and $\alpha_{2}$. The average deviation $\sigma$ was calculated using the same procedure. 


\section{Results and Discussion}

\subsection{Measurements Along the Arm}

Fig. 4 shows the results of the channel gain measurements along the arm at $55 \mathrm{GHz}$, $60 \mathrm{GHz}$, and $65 \mathrm{GHz}$, alongside the channel gain model described by Eq. 2, fitted to the data.

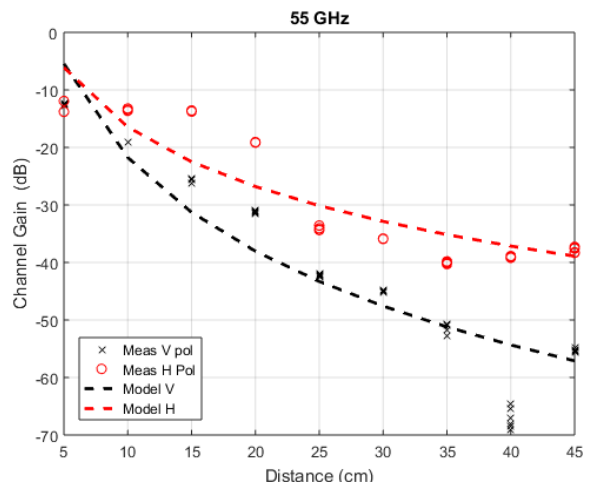

(a)

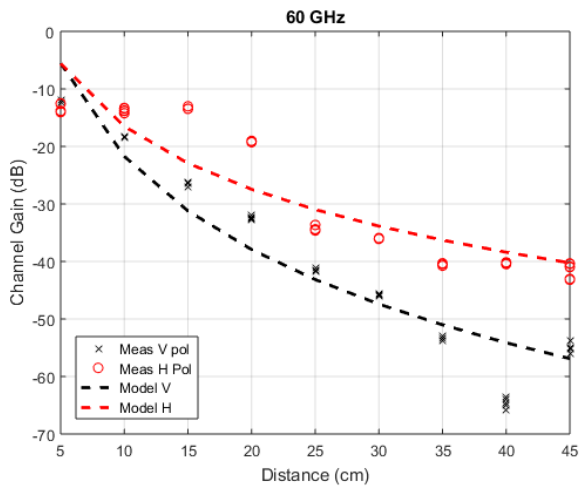

(b)

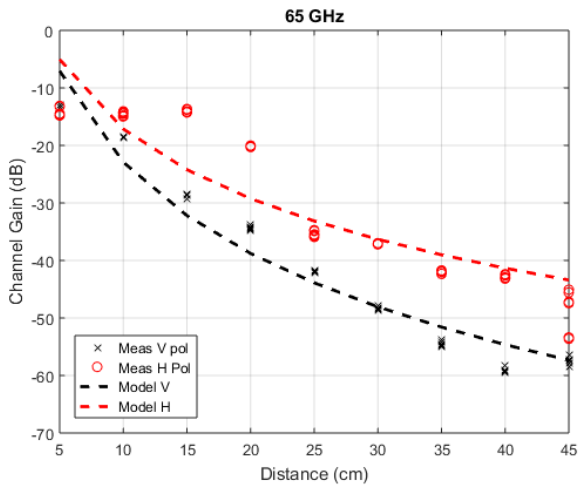

(c)

Fig. 4. Channel gain measurements along the arm of subject 1 in the configuration shown in Fig. 1 at (a) $55 \mathrm{GHz}$, (b) $60 \mathrm{GHz}$, and (c) $65 \mathrm{GHz}$.

The parameters of the channel gain model (Eq. 2) are shown in Table 1. 
Table 1. Parameters of the Channel Gain Model presented in Eq. 2.

\begin{tabular}{llllllll}
\hline \multicolumn{2}{l}{ Subject \& Frequency } & \multicolumn{2}{c}{$\mathrm{G}(10 \mathrm{~cm})(\mathrm{dB})$} & $\mathrm{n}$ & \multicolumn{2}{c}{$\sigma(d B)$} \\
\hline Subject 1 & & $\mathrm{H}$ & $\mathrm{V}$ & $\mathrm{H}$ & $\mathrm{V}$ & $\mathrm{H}$ & $\mathrm{V}$ \\
& & & & & & & \\
& $55 \mathrm{GHz}$ & -16 & -22 & 3.4 & 5.4 & 5.3 & 5.9 \\
& $60 \mathrm{GHz}$ & -17 & -22 & 3.6 & 5.4 & 5.5 & 5.1 \\
& $65 \mathrm{GHz}$ & -17 & -23 & 4.0 & 5.3 & 6.1 & 3.7 \\
Subject 2 & & & & & & & \\
& $55 \mathrm{GHz}$ & -17 & -22 & 4.7 & 4.4 & 3.6 & 3.2 \\
& $60 \mathrm{GHz}$ & -18 & -23 & 4.8 & 4.6 & 3.7 & 3.7 \\
& $65 \mathrm{GHz}$ & -18 & -23 & 4.7 & 4.5 & 4 & 3.8 \\
Subject 3 & & & & & & & 4.3 \\
& $55 \mathrm{GHz}$ & -19 & -24 & 5.1 & 5.3 & 4.3 & 4.3 \\
& $60 \mathrm{GHz}$ & -19 & -25 & 5.1 & 5.5 & 4.4 & 4.5 \\
& $65 \mathrm{GHz}$ & -19 & -25 & 5 & 5.7 & 4.6 & 5.4 \\
\hline
\end{tabular}

The channel gain exponents listed in Table 1 are between 3.4 and 5.7, while channel gains at $10 \mathrm{~cm}$ were found in a -25 to $-16 \mathrm{~dB}$ range. The parameters for the different subjects were found to be comparable. We found lower channel gain exponents for the $\mathrm{H}$-polarized antennas in comparison to $\mathrm{V}$-polarized antennas for subjects 1 and 3, while for subject 2, the channel loss exponent was slightly higher for H-polarization. This might be due to the higher body mass index (BMI) of subject 2 w.r.t. BMI of subjects 1 and 3 . The channel gain at $10 \mathrm{~cm}$ decreased with frequency. This was expected as larger relative distances in comparison to the wavelength had to be covered at higher frequencies (smaller wavelengths), which implies a lower channel gain [8]. Our results demonstrate a good agreement with literature. For instance [8] reported channel gain exponents from 3.5 up to 4 for a $60 \mathrm{GHz}$ dipole above a human skin phantom. The theory presented in [8] does predict a path loss exponent of exactly $n=4$. Most of the values we found were higher than 4 , which indicates that probably there were some near field data (exponent $>4$ ) included in the measurements. In [19], a pathloss exponent of 3.6 was reported for two rectangular waveguides at $60 \mathrm{GHz}$ on a skin phantom. In addition, [19] showed that different textiles have negligible effect on the channel gain at $60 \mathrm{GHz}$, which suggests that our measurements are usable for situations in which the arm is covered by clothing. The variation on the path loss $(\sigma)$ is around $5 \mathrm{~dB}$. For the horizontally polarized antennas on each of the three subjects the $\sigma$ slightly increases with increasing the frequency. Similar behavior is observed for vertically polarized horns (except subject 1).

In comparison to literature at lower frequencies, the channel gain exponents found in this study were generally higher. Channel gain along the arm was studied from 0.45 $2.4 \mathrm{GHz}$ in [16] for separation distances from $10-50 \mathrm{~cm}$ using the same model as presented in Eq. 2, resulting in channel gain exponents between 0.6 and 3.2. The values 
in [16] were obtained for indoor environments where reflections were present. This generally leads to lower path loss exponents [3]. In the contrary, in this study a very low amount of reflections was expected due to the higher frequencies, which would result in higher channel gain exponents. The IEEE channel gain model [3] valid at 0.4 - $11 \mathrm{GHz}$ found channel gain exponents from $0.5-2$ in indoor environments and 1.7 4.5 in anechoic conditions. The highest channel gain exponents in [3] were found between 3-11 GHz. [20] measured channel gain at $2.45 \mathrm{GHz}$ using dipoles in the H-polarization on the arm from $5-30 \mathrm{~cm}$ and found a channel gain exponent of 3.4 .

\subsection{Measurements Around the Arm}

Fig. 5 shows the channel gain as a function of the rotation around the wrist of the $\mathrm{TX}$ at $10 \mathrm{~cm}$ from the RX antenna.

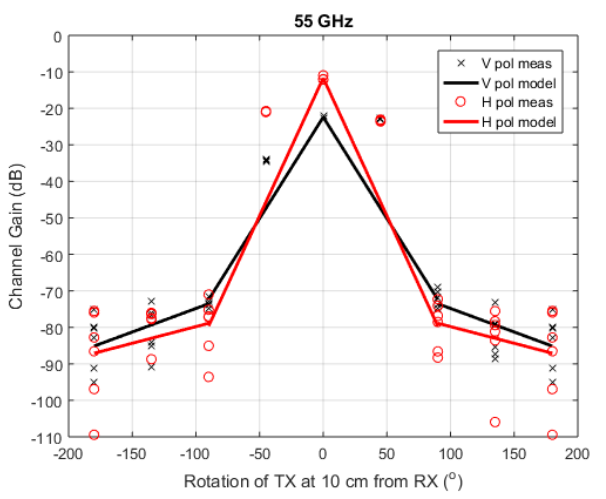

(a)

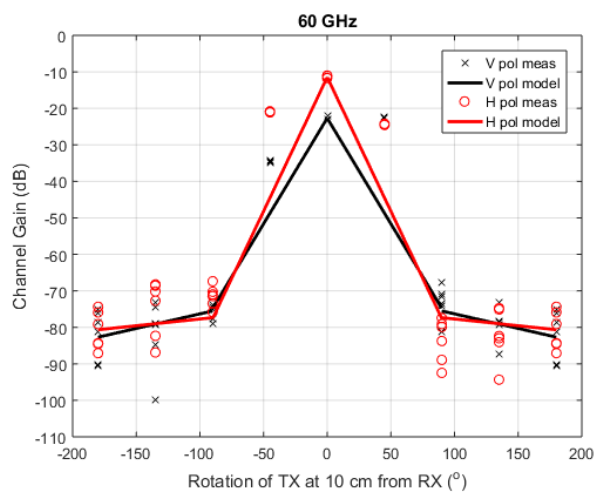

(b)

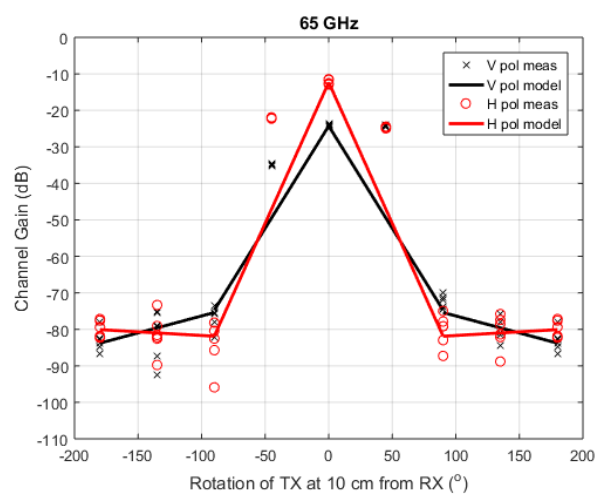

(c)

Fig. 5. Channel gain measurements for rotation of the TX around the arm of subject 1 in the configuration shown in Fig. 1 at (a) $55 \mathrm{GHz}$, (b) $60 \mathrm{GHz}$, and (c) $65 \mathrm{GHz}$. 
The parameters of the channel gain model shown in Eq. 5 are shown in Table II.

Table II. Parameters of the channel gain model in Eq. 5 fitted to measurements on subject 1.

\begin{tabular}{ccccccc}
\hline Frequency & \multicolumn{2}{c}{$\alpha_{1}\left(d B /^{\circ}\right)$} & \multicolumn{2}{c}{$\alpha_{2}\left(d B /^{\circ}\right)$} & \multicolumn{3}{c}{$\sigma(d B)$} \\
\hline & $\mathrm{H}$ & $\mathrm{V}$ & $\mathrm{H}$ & $\mathrm{V}$ & $\mathrm{H}$ & $\mathrm{V}$ \\
$55 \mathrm{GHz}$ & 0.76 & 0.56 & 0.091 & 0.13 & 7.5 & 4.6 \\
$60 \mathrm{GHz}$ & 0.73 & 0.58 & 0.037 & 0.080 & 5.2 & 2.8 \\
$65 \mathrm{GHz}$ & 0.77 & 0.56 & -0.020 & 0.093 & 2.6 & 1.6 \\
\hline
\end{tabular}

The loss per angle listed in Table II ranges from -0.02 to $0.77 \mathrm{~dB} /$ degree. The loss per degree was significantly higher for the angles $|\theta|<90^{\circ}\left(\alpha_{1}\right)$ in comparison to $|\theta|>$ $90^{\circ}\left(\alpha_{2}\right)$ as expected in Section 2.1. This justified our choice of $\theta_{c}=90^{\circ}$ and was also in line with the results found in [15] at lower frequencies for propagation around the human body. The $\mathrm{V}$-polarization had lower losses $\alpha_{1}$ for rotation angles $<90^{\circ}$ in comparison to H-polarization. It seems that since H-polarization is parallel to the arm, rotating the antennas results in a higher diffraction loss around the arm. On the contrary, the losses per angle $\alpha_{2}$ are higher for $\mathrm{V}$-polarization in comparison to H-polarization. Potentially, a better fit could be obtained by using a three-slope model instead of a twoslope model. Most of the loss (on a logarithmic scale) occurred between $45^{\circ}$ and $90^{\circ}$, which corresponded well with Eq. 3. Measurements with more angular resolution should be executed to determine this.

The losses per angle $\alpha_{1}$ for $|\theta|<90^{\circ}$ were found to be relatively large and would make communication with a single antenna at $60 \mathrm{GHz}$ around the $\operatorname{arm}\left(|\theta|>90^{\circ}\right)$ rather difficult, since we measured 50-70 dB loss over $90^{\circ}$ rotation. However, this opens up opportunities for good signal-to-interference ratios for channels that are $90^{\circ}$ rotated towards one another on the arm (assuming similar path gains as both TX and RX would rotate around the arm). An important note here is that the used antennas have a high gain $(19 \mathrm{dBi}$ at $60 \mathrm{GHz})$ and narrow radiation pattern $\left(3 \mathrm{~dB}\right.$ beam width of $14^{\circ}$ to $\left.24^{\circ}\right)$ in comparison to what can be expected from smaller on-body antennas.

\section{Conclusion}

Propagation of electromagnetic waves at frequencies from 55 to $65 \mathrm{GHz}$ was studied in a wrist-to-arm scenario. To this aim antennas tuned to that frequency band were placed on the left arm of three subjects in two measurement scenarios that are representative for communication from a wristband to an arm-worn sensor: (1) transmission along the arm and (2) communication between a rotated transmitter and a fixed receiver. A log-linear model, normalized to the channel gain at $10 \mathrm{~cm}$ antenna separation, was fitted to the measured scattering parameters obtained in the first scenario, resulting in an estimation of channel gain exponents. A dual-slope model was fitted to the data obtained in the second scenario (rotation scenario) in order to obtain two loss factors 
that describe the loss in channel gain per rotational angle around the wrist. In the along the arm scenario, channel gain exponents between 3.4 and 5.7 were obtained, in comparison to a theoretically expected value of 4 . The channel gains at $10 \mathrm{~cm}$ ranged from -25 to $-16 \mathrm{~dB}$. The measured losses per angle in the second scenario ranged from -0.02 to $0.77 \mathrm{~dB} /$ degree with significantly lower losses for rotational angles beyond $90^{\circ}$. The loss per angle for rotation from $0^{\circ}$ to $90^{\circ}$ was found to be higher than $0.56 \mathrm{~dB} /{ }^{\circ}$, while this quantity is smaller than $0.13 \mathrm{~dB} /{ }^{\circ}$ for additional rotations beyond $90^{\circ}$ up to $270^{\circ}$. This suggests the potential for simultaneous operation of multiple wireless channels in the studied frequency band on the same arm with favorable signal-to-interference ratios.

\section{Acknowledgment}

A. Thielens is a post-doctoral Fellow of Flanders Innovation and Entrepreneurship under grant No. 150752. A.T. has received funding from the European Union's Horizon 2020 research and innovation programme under the Marie Skłodowska-Curie grant agreement No 665501 with the research Foundation Flanders (FWO). A.T. is an FWO [PEGASUS] $]^{2}$ Marie Skłodowska-Curie Fellow.

\section{References}

1. M. Chen, S. Gonzalez, A. Vasilakos, H. Cao, V.C. Leung, "Body area networks: A survey," Mobile Netw. Appl., vol. 16, pp. 171-193, 2011.

2. M. Ghamari, B. Janko, R.S. Sherratt, W. Harwin, R. Piechockic, C. Soltanpur, "A survey on wireless body area networks for ehealthcare systems in residential environments," Sensors, vol. 16, no.831, 2016.

3. IEEE, P802.15 Working Group for Wireless Personal Area Networks (WPANs), "Channel Model for Body Area Network (BAN),” IEEE P802.15-08-0780-09-0006. April 2009.

4. ITU standard

5. Pellegrini et al., "Antennas and Propagation for Body-Centric Wireless Communications at Millimeter-Wave Frequencies: A Review", IEEE Antennas and Propagation magazine 55(4): 262-287, 2013.

6. N. Chahat, M. Zhadobov, L. Le Coq and R. Sauleau, "Wearable Endfire Textile Antenna for On-Body Communications at $60 \mathrm{GHz}$," IEEE Antennas and Wireless Propagation Letters, 11, 2012, pp. 799-802.

7. N. Chahat, M. Zhadobov, and R. Sauleau, "60-GHz Textile Antenna Array for Body-Centric Communications," IEEE Transactions on Antennas Propagation, AP-61, 4, April 2013, pp. 1816-1824.

8. N. Chahat, G. Valerio, M. Zhadobov and R. Sauleau, "On- Body Propagation at $60 \mathrm{GHz}$," IEEE Transactions on Antennas and Propagation, AP-61, 4, April 2013, pp. 1876-188.

9. Hall et al., "Advances in Antennas and Propagation for Body Centric Wireless Communications", Proceedings of the Fourth European Conference on Antennas and Propagation, 1216 April 2010, Barcelona, Spain, 2010.

10. Luca Petrillo, Theodoros Mavridis, Julien Sarrazin, Jean-Michel Dricot, Aziz Benlarbi-Delai, et al. "BAN Working Frequency: a Trade-Off Between Antenna Efficiency and Propagation Losses”. Conference EuCAP 2014, Apr 2014, La Haye, Netherlands. 2014. 
11. K. A. Norton, "The propagation of radio waves over the surface of the earth and in the upper atmosphere-Part I Ground-wave propagation from short antennas," Proc. IRE, vol. 24, no. 10, pp. 1367-1387, Oct. 1936.

12. J. Bae, H. cho, K. Song, H. Lee, and H.-J. Yoo, "The Signal Transmission Mechanism on the Surface of Human Body for Body Channel Communication”, IEEE Trans. MTT 60(3), pp. 582-593, 2012.

13. C.A. Balanis, "Antenna Theory", Wiley, 1984.

14. A. Fort, F. Keshmiri, G. R. Crusats, C. Craeye, C. Oestges, “A Body Area Propagation Model Derived from Fundamental Principles: Analytical Analysis and Comparison with Measurements", IEEE Trans A\&P 58(2), pp. 503-513, 2010.

15. J. Ryckaert, P. De Doncker, R. Meys, A. De Le Hoye, S. Donnay, "Channel model for wireless communication around human body", Electronics Letter Vol. 40 No. 9, pp. 543 - 544, 2004.

16. A. Thielens, R. Benarrouch, S. Wielandt, M.G. Anderson, A. Moin, A. Cathelin, J. Rabaey. "A Comparative Study of On-Body Radio-Frequency Links in the $420 \mathrm{MHz}-2.4 \mathrm{GHz}$ Range". Sensors 18(12): E4165, 2018.

17. Anderson, M.G.; Thielens, A.; Wielandt, S.; Niknejad, A.; Rabaey, J.M, "Ultra-Low Power On-Antenna Beamforming for Antenna Arrays Using Tunable Passives", IEEE MTT Letters 29(2) pp. 158-160, 2019.

18. Marinova, M.; Thielens, A.; Tanghe, E.; Vallozzi, L.; Vermeeren, G.; Joseph, W.; Rogier, H.; Martens, L. "Diversity performance of off-body MB-OFDM UWB-MIMO". IEEE Transactions on Antennas and Propagation 2015, 63, 3187-3197.

19. A. R. Guraliuc, M. Zhadobov, G. Valerio, N. Chahat, and R. Sauleau, "Effect of Textile on the Propagation Along the Body at $60 \mathrm{GHz}$," IEEE Transactions on Antennas and Propagation, vol. 62, no. 3, pp. 1489-1494, Mar. 2014.

20. E. Reussens, W. Joseph, B. Latré, G. Vermeeren, E.Tanghe, L. Martens, I. Moereman, C. Blondia, "Characterization of On-Body Communication Channel and Energy Efficient Topology Design for Wireless Body Area Networks", IEEE Trans Inf. Tech. Biomed. 13(6), pp. $933-945,2009$. 